\title{
Risk of Dry Eye Syndrome in Patients with Orbital Fracture: A Nationwide Population-Based Cohort Study
}

\author{
Cindy Yi-Yu Hsu ${ }^{1}$, Junior Chun-Yu Tu ${ }^{2}$, Chi-Hsiang Chung ${ }^{3,4,5}{ }^{(}$, Chien-An Sun $\left.{ }^{6,7}{ }^{(}\right)$, Wu-Chien Chien ${ }^{3,4,5,8, *}$ \\ and Hsin-Ting Lin ${ }^{9,10, *}$
}

check for updates

Citation: Hsu, C.Y.-Y.; Tu, J.C.-Y.; Chung, C.-H.; Sun, C.-A.; Chien, W.-C.; Lin, H.-T. Risk of Dry Eye Syndrome in Patients with Orbital Fracture: A Nationwide Population-Based Cohort Study. Healthcare 2021, 9, 605. https:// doi.org/10.3390/healthcare9050605

Academic Editors: Pedram Sendi and Flavia Leao Barbosa

Received: 11 April 2021

Accepted: 14 May 2021

Published: 18 May 2021

Publisher's Note: MDPI stays neutral with regard to jurisdictional claims in published maps and institutional affiliations.

Copyright: (C) 2021 by the authors Licensee MDPI, Basel, Switzerland. This article is an open access article distributed under the terms and conditions of the Creative Commons Attribution (CC BY) license (https:// creativecommons.org/licenses/by/ $4.0 /)$.
1 Department of Ophthalmology, Mackay Memorial Hospital, Taipei 104, Taiwan; yiyu0610@gmail.com

2 Department of Plastic Surgery, Chang Gung Memorial Hospital, Chung Gung Medical University, Taoyuan 333, Taiwan; jr_tu@hotmail.com

3 School of Public Health, National Defense Medical Center, Taipei 114, Taiwan; g694810042@gmail.com

4 Department of Medical Research, Tri-Service General Hospital, National Defense Medical Center, Taipei 114, Taiwan

5 Taiwanese Injury Prevention and Safety Promotion Association (TIPSPA), Taipei 114, Taiwan

6 Department of Public Health, College of Medicine, Fu-Jen Catholic University, New Taipei City 242, Taiwan; 040866@mail.fju.edu.tw

7 Big Data Research Center, College of Medicine, Fu-Jen Catholic University, New Taipei City 242, Taiwan

8 Graduate Institute of Life Sciences, National Defense Medical Center, Taipei 114, Taiwan

9 Department of Ophthalmology, Tri-Service General Hospital, National Defense Medical Center, Taipei 114, Taiwan

10 Graduate Institute of Medical Sciences, National Defense Medical Center, Taipei 114, Taiwan

* Correspondence: chienwu@ndmctsgh.edu.tw (W.-C.C.); $808010005 @$ mail.ndmctsgh.edu.tw (H.-T.L.); Tel.: +886-2-87923311 (ext. 19189) (W.-C.C.); +886-2-87923311 (H.-T.L.); Fax: +886-2-87927235 (W.-C.C.)

Abstract: This study aimed to investigate whether orbital fracture increases the risk of dry eye syndrome (DES) and identified the profile of prognostic factors. We studied a cohort from the Taiwan National Health Insurance Research Database (NHIRD). Overall, 46,179 and 184,716 participants were enrolled in the study and control groups, respectively. Each patient in the case group was ageand gender-matched to four individuals without orbital fracture that served as the control group. Cox proportional hazards analysis regression was used to estimate the risks of incident DES. During the follow-up period, the case group was more likely to develop incident DES $(0.17 \%)$ than the control group $(0.11 \%)(p=0.001)$. Multivariate Cox regression analysis demonstrated that the case group had a 4.917-fold increased risk of DES compared to the controls. In the stratified age group, orbital fracture had the highest impact on patients aged 18-29 years. Furthermore, patients with orbital roof fracture have a greater risk of developing DES. Regardless of whether having received surgery or not, the patients with orbital fracture have higher risks of DES. Our study demonstrated that orbital fracture increases the risk of developing subsequent DES. Early recognition by thorough examinations with raised awareness in the clinical setting could preserve visual function and prevent further complications.

Keywords: dry eye syndrome; orbital fracture; ocular surface

\section{Introduction}

The orbit is a bony cavity that contains the globe, extraocular muscles, nerves, fat, and blood vessels. Blunt trauma to the orbital rim leads to orbital fractures and causes damages to the surrounding facial bones and soft tissues [1-3]. Trauma to the eye and surrounding parts accounts for approximately 3\% of all emergency department visits in the United States [4]. The predominant etiology of injury was violence (physical assault) followed by traffic accidents and sports injuries [5-8]. Males in their thirties are the most susceptible population $[3,6,7,9]$. The orbital fracture is often described according to the location of the injury, such as the floor, roof, medial wall, and lateral wall, with the orbital floor as 
the most common isolated orbital bone fracture site [6,8]. Additionally, a systemic review study found that 43 patients among 532 orbital fractures $(8.1 \%)$ have led to a decrease in visual acuity [10].

To what extent the orbital trauma will affect ocular tear film stability is unclear [11]. Based on clinical experience, we have hypothesized that orbital fracture might be related to subsequent dry eye syndrome (DES). There has yet to be a large population study to support this hypothesis. Therefore, we conducted a longitudinal nationwide population-based cohort study using the Taiwan National Health Insurance Research Database (NHIRD).

DES is a multifactorial disease of the tears and the ocular surface that results in symptoms of discomfort, visual disturbance, and tear film instability with potential damage to the ocular surface. It is accompanied by increased osmolarity of the tear film and the inflammation of the ocular surface. A decrease in visual acuity associated with daily acts of gazing has been proven in dry eye patients [12]. The mechanism is related to disrupted tear film causing ocular surface irregularity [12-14]. DES increases with age and the prevalence is higher in women compared to men. The odds for DES increase 35\% for each additional 10 years of age and the odds also increase for women [15]. Several independent risk factors have been found to be associated with DES: diabetes, connective tissue disease, hepatitis C, total to high-density lipoprotein cholesterol ratio, postmenopausal estrogen therapy, antihistamines, antidepressants, smoking status, caffeine use, contact lenses, and video display terminal exposure for more than $6 \mathrm{~h}$ /day $[15,16]$. Therefore, DES is considered a complex multi-factorial disease.

This study aimed to investigate whether orbital fracture increases the risk of DES. Moreover, potential risk factors, including several diseases and medications that may induce DES, were analyzed in the multivariable model. Furthermore, we discussed the association between surgery treatment for orbital fracture and DES.

\section{Method}

\subsection{Data Resource and Ethics Declaration}

The claims data used in the current study were accessed from the 2005 Longitudinal Health Insurance Database (LHID), which was derived from the NHIRD. The LHID was a subset of the NHIRD. It contained information from 2 million people and was used in the present study that randomly sampled individuals between 2000 and 2015. There was no significant difference in the distribution of sex, age, and insured premium between the LHID and the original NHIRD. Taiwan has initiated the National Health Insurance program in 1995. It covers approximately 99\% of Taiwan's population [17]. The data of LHID was randomly sampled from the NHIRD registry for the year 2005 by the database of the National Health Insurance Administration. The information available from the LHID include the demographic data of the subjects, their socioeconomic conditions, the residence of the subjects, the International Classification of Diseases-Ninth Revision (ICD9), the International Classification of Diseases-Tenth Revision (ICD-10), and the medications used by each of the study subjects. The accuracy and high validity of diagnoses in the NHIRD have been demonstrated in previous articles [18]. The time interval of LHID ranges from 1 January 2000 to 31 December 2015, with a total study interval of about 15 years. This retrospective, population-based cohort study was approved by both the National Health Insurance Administration and the Institutional Review Board of Tri-Service General Hospital (TSGHIRB No. B-110-02). In addition, the need for informed consent was waived by the two institutions.

\subsection{Study Participants}

The flowchart of study sample selection from the LHID is shown in Figure 1. Of the total sample, 47,326 patients were followed up at the outpatient department more than 2 times with the diagnosis of orbital fracture or were hospitalized with orbital fracture being one of the, if not the only, diagnosis. The orbital fracture diagnostic codes include orbital floor closed fracture (ICD-9-CM code, 802.6), orbital floor open fracture (ICD-9-CM code, 
8027), orbital roof closed fracture (ICD-9-CM code, 801.0 801.4), and orbital roof open fracture (ICD-9-CM code, 801.5 801.9). The index date was defined as the date of newly diagnosed orbital fracture. Furthermore, the following exclusion criteria were applied: being diagnosed with DES before the index date; being diagnosed with orbital fracture before the index date; having a diagnosis of severe ocular trauma at any time; having received eyeball removal surgery before the index date; without tracking; age $<18$ years; and gender unknown. Finally, 46,179 patients matched our criteria and were assigned to the study cohort. For each orbital fracture patient, the four comparisons (1:4) were frequency matched by age (each 5-year span), sex, and index date as the control cohort.

\section{Outpatient and inpatient of Longitudinal Health Insurance Database in 2000-2015 in Taiwan}

$$
n=1936512
$$

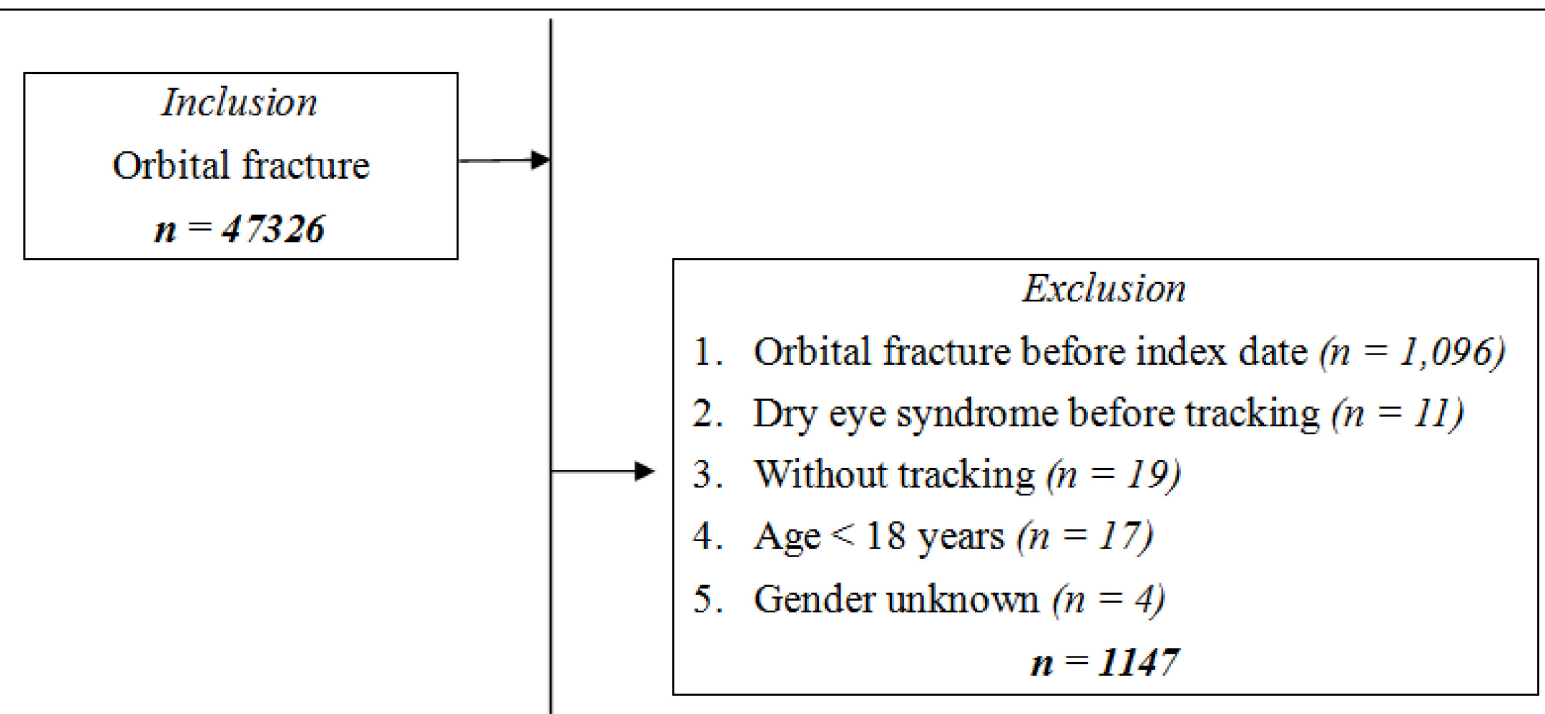

1. Without orbital fracture in study period

2. The same exclusion criteria

3. 4-fold propensity score matching by gender, age, and index date

\begin{tabular}{|c|c|}
\hline $\begin{array}{c}\text { With orbital fracture } \\
\text { (Study cohort) } \\
\boldsymbol{n}=\mathbf{4 6 1 7 9} \\
\downarrow\end{array} \mid \begin{array}{c}\text { Without orbital fracture } \\
\text { (Comparison cohort) } \\
\boldsymbol{n}=\mathbf{1 8 4 7 1 6}\end{array}$ \\
\hline Dry eye syndrome \\
$\boldsymbol{n}=\mathbf{7 9}$
\end{tabular}

Figure 1. Flowchart of the study sample selection.

\subsection{Main Outcome Measurement}

The development of DES was defined as the main outcome in the current study, which was based on the DES diagnosis (ICD-9-CM code, 375.15) after the index date. Moreover, only those patients having received a diagnosis of DES by an ophthalmologist were considered as having achieved the primary outcome and included in this study. In clinical practice, ICD-9/ICD-10 codes for "unspecific corneal disorder" may also be used 
for some forms of dry eye disease. However, these codes were eliminated to prevent overestimation and confusion of the primary outcome.

\subsection{Demographic Variables and Comorbidities}

To standardize the health condition of participants and to compare the baseline characteristics between the two groups, we considered the effects of demographic conditions including age, gender, urbanization level, income level, and the following comorbidities in the analysis: hypertension, diabetes mellitus, hyperlipidemia, ischemic heart diseases, congestive heart failure, chronic obstructive pulmonary disease, liver disease, and rheumatic disease. Moreover, in this study, we included several common factors of DES, such as connective tissue disease, multiple sclerosis (MS), osteoporosis, Bell's palsy, and Parkinson's disease to evaluate the confounding effects of orbital fracture. Additionally, to make the ocular condition of the study population more homogenous, we included the effect of trachoma, blepharitis, hordeolum, and glaucoma in the multivariable model. We then longitudinally followed the patients' condition from the index date until the date of DES diagnosis or until the last date of data collection from the LHID, which is 31 December 2015.

\subsection{Statistical Analysis}

Statistical Product and Service Solutions (SPSS) 22nd edition (Armonk, NY, USA: IBM Corp.) was used for all the statistical analyses in the current study. The demographic features and common comorbidities between the orbital fracture patients and the control cohort were compared using the Chi-square test or Fisher's exact test. The mean ages (continuous data) of both cohorts were measured using Student's $t$-test. Then, the Cox proportional hazard regression was adopted to yield adjusted hazard ratios (aHR) of DES by incorporating the above demographic data, ocular diseases, and systemic comorbidities in the multivariable analysis. The incidence rate (per 105 person-years) of DES was calculated according to sex, age, and comorbidities for each cohort. For the subgroup analysis, the sensitivity analysis with aHR of DES that stratified by the surgery or not and orbital fracture types were conducted. In addition, the cumulative risks of DES were calculated with the Kaplan-Meier method and compared by log-rank test. Two-tailed $p$-values $<0.05$ was considered as statistically significant.

\section{Result}

\subsection{Sample Characteristics}

The baseline demographic characteristics and common comorbidities of the case group and control group are shown in Table 1 . The present study included 46,179 patients with newly diagnosed orbital fracture and 184,716 patients in the control cohort between 2000 and 2015. No significant differences were noted between the patients and controls in sex and age distribution at baseline. Of the patients with orbital fracture, 33,115 $(71.71 \%)$ were males, and 15,295 (33.12\%) were aged 18-29 years (mean age, $42.32 \pm 17.99$ years). The case group was more likely to have low insured premium, residence in higher urbanized areas, and high health care level at the time of the index date. Lower prevalence of most concomitant comorbidities (except Bell's palsy) was noted in the case group. The prevalence of trachoma, blepharitis, and hordeolum had no significant difference between the two groups. 
Table 1. Characteristics of study in the baseline.

\begin{tabular}{|c|c|c|c|c|c|}
\hline \multirow{2}{*}{$\begin{array}{l}\text { Orbital Fracture } \\
\text { Variables }\end{array}$} & \multicolumn{2}{|c|}{ With } & \multicolumn{2}{|c|}{ Without } & \multirow{2}{*}{$p$} \\
\hline & $n$ & $\%$ & $n$ & $\%$ & \\
\hline Total & 46,179 & 20.00 & 184,716 & 80.00 & \\
\hline Gender & & & & & 0.999 \\
\hline Male & 33,115 & 71.71 & 132,460 & 71.71 & \\
\hline Female & 13,064 & 28.29 & 52,256 & 28.29 & \\
\hline Age (years) & \multicolumn{2}{|c|}{$42.32 \pm 17.99$} & \multicolumn{2}{|c|}{$42.38 \pm 17.81$} & 0.518 \\
\hline \multicolumn{3}{|l|}{ Age group (yrs) } & & & 0.999 \\
\hline $18-29$ & 15,295 & 33.12 & 61,180 & 33.12 & \\
\hline $30-39$ & 7962 & 17.24 & 31,848 & 17.24 & \\
\hline $40-49$ & 7511 & 16.26 & 30,044 & 16.26 & \\
\hline $50-59$ & 6470 & 14.01 & 25,880 & 14.01 & \\
\hline$\geq 60$ & 8941 & 19.36 & 35,764 & 19.36 & \\
\hline \multicolumn{3}{|l|}{ Insured premium (NT\$) } & & & $<0.001$ \\
\hline$<18,000$ & 45,279 & 98.05 & 180,980 & 97.98 & \\
\hline $18,000-34,999$ & 699 & 1.51 & 2606 & 1.41 & \\
\hline$\geq 35,000$ & 201 & 0.44 & 1130 & 0.61 & \\
\hline \multicolumn{3}{|l|}{ Education levels (yrs) } & & & 0.337 \\
\hline$<12$ & 23,792 & 51.52 & 95,629 & 51.77 & \\
\hline$\geq 12$ & 22,387 & 48.48 & 89,087 & 48.23 & \\
\hline Diabetes mellitus (DM) & 5862 & 12.69 & 33,957 & 18.38 & $<0.001$ \\
\hline Hyperlipidemia & 1562 & 3.38 & 16,416 & 8.89 & $<0.001$ \\
\hline Hypertension (HTN) & 8487 & 18.38 & 49,378 & 26.73 & $<0.001$ \\
\hline Chronic kidney disease (CKD) & 1900 & 4.11 & 20,174 & 10.92 & $<0.001$ \\
\hline Coronary artery disease (CAD) & 3428 & 7.42 & 32,220 & 17.44 & $<0.001$ \\
\hline Congestive heart failure (CHF) & 1293 & 2.80 & 13,204 & 7.15 & $<0.001$ \\
\hline Stroke & 4408 & 9.55 & 25,696 & 13.91 & $<0.001$ \\
\hline Chronic obstructive pulmonary disease (COPD) & 2803 & 6.07 & 24,213 & 13.11 & $<0.001$ \\
\hline Chronic liver disease (CLD) & 2847 & 6.17 & 29,364 & 15.90 & $<0.001$ \\
\hline Osteoporosis & 239 & 0.52 & 3065 & 1.66 & $<0.001$ \\
\hline Rheumatoid arthritis (RA) & 104 & 0.23 & 986 & 0.53 & $<0.001$ \\
\hline Connective tissue disease (CTD) & 98 & 0.21 & 807 & 0.44 & $<0.001$ \\
\hline Sarcoidosis & 4 & 0.01 & 30 & 0.02 & 0.287 \\
\hline Trachoma & 74 & 0.16 & 242 & 0.13 & 0.137 \\
\hline Multiple sclerosis (MS) & 8 & 0.02 & 90 & 0.05 & 0.004 \\
\hline Bell's palsy & 896 & 1.94 & 569 & 0.31 & $<0.001$ \\
\hline Parkinson disease & 468 & 1.01 & 2900 & 1.57 & $<0.001$ \\
\hline Blepharitis & 17 & 0.04 & 109 & 0.06 & 0.079 \\
\hline Hordeolum & 29 & 0.06 & 177 & 0.10 & 0.039 \\
\hline Glaucoma & 254 & 0.55 & 1354 & 0.73 & $<0.001$ \\
\hline CCI_R & \multicolumn{2}{|c|}{$0.04 \pm 0.39$} & \multicolumn{2}{|c|}{$0.31 \pm 1.34$} & $<0.001$ \\
\hline Anti-HTN drugs & 7923 & 17.16 & 41,356 & 22.39 & $<0.001$ \\
\hline Antidepressants drugs & 2656 & 5.75 & 9752 & 5.28 & $<0.001$ \\
\hline Anti-CA drugs & 136 & 0.29 & 555 & 0.30 & 0.834 \\
\hline Anti-Parkinson drugs & 302 & 0.65 & 1915 & 1.04 & $<0.001$ \\
\hline Anti-ulcer drugs & 131 & 0.28 & 228 & 0.12 & $<0.001$ \\
\hline Muscle spasm drugs & 206 & 0.45 & 701 & 0.38 & 0.041 \\
\hline Decongestant drugs & 389 & 0.84 & 1315 & 0.71 & 0.003 \\
\hline Antihistamines drugs & 1136 & 2.46 & 4499 & 2.44 & 0.762 \\
\hline Anesthetics drugs & 117 & 0.25 & 430 & 0.23 & 0.416 \\
\hline \multicolumn{5}{|l|}{ Season } & 0.999 \\
\hline Spring (Mar-May) & 11,178 & 24.21 & 44,712 & 24.21 & \\
\hline Summer (Jun-Aug) & 11,339 & 24.55 & 45,356 & 24.55 & \\
\hline Autumn (Sep-Nov) & 12,069 & 26.14 & 48,276 & 26.14 & \\
\hline Winter (Dec-Feb) & 11,593 & 25.10 & 46,372 & 25.10 & \\
\hline
\end{tabular}


Table 1. Cont.

\begin{tabular}{|c|c|c|c|c|c|}
\hline \multirow{2}{*}{$\begin{array}{c}\text { Orbital Fracture } \\
\text { Variables }\end{array}$} & \multicolumn{2}{|c|}{ With } & \multicolumn{2}{|c|}{ Without } & \multirow{2}{*}{$p$} \\
\hline & $n$ & $\%$ & $n$ & $\%$ & \\
\hline Location & & & & & $<0.001$ \\
\hline Northern Taiwan & 15,789 & 34.19 & 74,088 & 40.11 & \\
\hline Middle Taiwan & 12,434 & 26.93 & 51,859 & 28.07 & \\
\hline Southern Taiwan & 14,925 & 32.32 & 47,312 & 25.61 & \\
\hline Eastern Taiwan & 2865 & 6.20 & 10,583 & 5.73 & \\
\hline Outlets islands & 166 & 0.36 & 874 & 0.47 & \\
\hline Urbanization level & & & & & $<0.001$ \\
\hline 1 (The highest) & 16,440 & 35.60 & 63,131 & 34.18 & \\
\hline 2 & 19,802 & 42.88 & 76,262 & 41.29 & \\
\hline 3 & 3440 & 7.45 & 16,404 & 8.88 & \\
\hline 4 (The lowest) & 6497 & 14.07 & 28,919 & 15.66 & \\
\hline Level of care & & & & & $<0.001$ \\
\hline Hospital center & 19,913 & 43.12 & 54,086 & 29.28 & \\
\hline Regional hospital & 20,770 & 44.98 & 55,894 & 30.26 & \\
\hline Local hospital & 5496 & 11.90 & 74,736 & 40.46 & \\
\hline
\end{tabular}

$p$ : Chi-square/Fisher exact test on category variables and $t$-test on continue variables.

The distributions of incident DES and related clinical manifestations for the two groups during the 15-year follow-up are presented in Table 2. Compared with the control group $(0.11 \%)$, the orbital fracture cohort had a higher incidence of DES $(0.17 \%)$ during the follow-up period $(p=0.001)$. The average ages were $45.72 \pm 19.04$ and $49.11 \pm 19.25$ years for the study and the comparison cohort, respectively $(p<0.001)$. The mean follow-up time was $10.29 \pm 15.95$ years in the orbital fracture cohort and $10.75 \pm 9.91$ years in the control cohort (Supplementary Table S1). The mean duration to develop DES in the orbital fracture cohort was $4.13 \pm 2.84$ years, which is shorter than the control cohort (6.91 \pm 4.57 years; Supplementary Table S2).

Table 2. Characteristics of study in the endpoint.

\begin{tabular}{|c|c|c|c|c|c|}
\hline \multirow{2}{*}{$\begin{array}{c}\text { Orbital Fracture } \\
\text { Variables }\end{array}$} & \multicolumn{2}{|c|}{ With } & \multicolumn{2}{|c|}{ Without } & \multirow{2}{*}{$p$} \\
\hline & $n$ & $\%$ & $n$ & $\%$ & \\
\hline Total & 46,179 & 20 & 184,716 & 80 & \\
\hline Dry eye syndrome & & & & & 0.001 \\
\hline Without & 46,100 & 99.83 & 184,510 & 99.89 & \\
\hline With & 79 & 0.17 & 206 & 0.11 & \\
\hline Gender & & & & & 0.999 \\
\hline Male & 33,115 & 71.71 & 132,460 & 71.71 & \\
\hline Female & 13,064 & 28.29 & 52,256 & 28.29 & \\
\hline Age (years) & \multicolumn{2}{|c|}{$45.72 \pm 19.04$} & \multicolumn{2}{|c|}{$49.11 \pm 19.25$} & $<0.001$ \\
\hline Age group (yrs) & & & & & $<0.001$ \\
\hline $18-29$ & 12,414 & 26.88 & 35,791 & 19.38 & \\
\hline $30-39$ & 8152 & 17.65 & 37,077 & 20.07 & \\
\hline $40-49$ & 7360 & 15.94 & 30,221 & 16.36 & \\
\hline $50-59$ & 6801 & 14.73 & 25,180 & 13.63 & \\
\hline$\geq 60$ & 11,452 & 24.8 & 56,447 & 30.56 & \\
\hline Insured premium (NT\$) & & & & & $<0.001$ \\
\hline$<18,000$ & 45,279 & 98.05 & 180,980 & 97.98 & \\
\hline $18,000-34,999$ & 699 & 1.51 & 2606 & 1.41 & \\
\hline$\geq 35,000$ & 201 & 0.44 & 1130 & 0.61 & \\
\hline \multicolumn{6}{|l|}{ Education levels (yrs) } \\
\hline$<12$ & 46,179 & 100 & 184,716 & 100 & \\
\hline$\geq 12$ & & 0 & & 0 & \\
\hline
\end{tabular}


Table 2. Cont.

\begin{tabular}{|c|c|c|c|c|c|}
\hline \multirow{2}{*}{$\begin{array}{c}\text { Orbital Fracture } \\
\text { Variables }\end{array}$} & \multicolumn{2}{|c|}{ With } & \multicolumn{2}{|c|}{ Without } & \multirow{2}{*}{$p$} \\
\hline & $n$ & $\%$ & $n$ & $\%$ & \\
\hline $\mathrm{DM}$ & 5862 & 12.69 & 33,957 & 18.38 & $<0.001$ \\
\hline Hyperlipidemia & 1562 & 3.38 & 16,416 & 8.89 & $<0.001$ \\
\hline HTN & 8487 & 18.38 & 49,378 & 26.73 & $<0.001$ \\
\hline CKD & 1900 & 4.11 & 20,174 & 10.92 & $<0.001$ \\
\hline CAD & 3428 & 7.42 & 32,220 & 17.44 & $<0.001$ \\
\hline $\mathrm{CHF}$ & 1293 & 2.8 & 13,204 & 7.15 & $<0.001$ \\
\hline Stroke & 4408 & 9.55 & 25,696 & 13.91 & $<0.001$ \\
\hline COPD & 2803 & 6.07 & 24,213 & 13.11 & $<0.001$ \\
\hline CLD & 2847 & 6.17 & 29,364 & 15.9 & $<0.001$ \\
\hline Osteoporosis & 239 & 0.52 & 3065 & 1.66 & $<0.001$ \\
\hline RA & 104 & 0.23 & 986 & 0.53 & $<0.001$ \\
\hline CTD & 98 & 0.21 & 807 & 0.44 & $<0.001$ \\
\hline Sarcoidosis & 4 & 0.01 & 30 & 0.02 & 0.287 \\
\hline Trachoma & 74 & 0.16 & 242 & 0.13 & 0.137 \\
\hline MS & 8 & 0.02 & 90 & 0.05 & 0.004 \\
\hline Bell's palsy & 896 & 1.94 & 569 & 0.31 & $<0.001$ \\
\hline Parkinson disease & 468 & 1.01 & 2900 & 1.57 & $<0.001$ \\
\hline Blepharitis & 17 & 0.04 & 109 & 0.06 & 0.079 \\
\hline Hordeolum & 29 & 0.06 & 177 & 0.1 & 0.039 \\
\hline Glaucoma & 254 & 0.55 & 1354 & 0.73 & $<0.001$ \\
\hline CCI_R & \multicolumn{2}{|c|}{$0.04 \pm 0.39$} & \multicolumn{2}{|c|}{$0.31 \pm 1.34$} & $<0.001$ \\
\hline Anti-HTN drugs & 7923 & 17.16 & 41,356 & 22.39 & $<0.001$ \\
\hline Antidepressants drugs & 2656 & 5.75 & 9752 & 5.28 & $<0.001$ \\
\hline Anti-CA drugs & 136 & 0.29 & 555 & 0.3 & 0.834 \\
\hline Anti-Parkinson drugs & 302 & 0.65 & 1915 & 1.04 & $<0.001$ \\
\hline Anti-ulcer drugs & 131 & 0.28 & 228 & 0.12 & $<0.001$ \\
\hline Muscle spasm drugs & 206 & 0.45 & 701 & 0.38 & 0.041 \\
\hline Decongestant drugs & 389 & 0.84 & 1315 & 0.71 & 0.003 \\
\hline Antihistamines drugs & 1136 & 2.46 & 4499 & 2.44 & 0.762 \\
\hline Anesthetics drugs & 117 & 0.25 & 430 & 0.23 & 0.416 \\
\hline Season & & & & & $<0.001$ \\
\hline Spring & 10,654 & 23.07 & 45,045 & 24.39 & \\
\hline Summer & 11,535 & 24.98 & 48,254 & 26.12 & \\
\hline Autumn & 12,846 & 27.82 & 47,521 & 25.73 & \\
\hline Winter & 11,144 & 24.13 & 43,896 & 23.76 & \\
\hline Location & & & & & $<0.001$ \\
\hline Northern Taiwan & 16,139 & 34.95 & 73,831 & 39.97 & \\
\hline Middle Taiwan & 12,431 & 26.92 & 52,105 & 28.21 & \\
\hline Southern Taiwan & 14,703 & 31.84 & 47,128 & 25.51 & \\
\hline Eastern Taiwan & 2740 & 5.93 & 10,798 & 5.85 & \\
\hline Outlets islands & 166 & 0.36 & 854 & 0.46 & \\
\hline Urbanization level & & & & & $<0.001$ \\
\hline 1 (The highest) & 15,777 & 34.16 & 60,659 & 32.84 & \\
\hline 2 & 19,613 & 42.47 & 78,985 & 42.76 & \\
\hline 3 & 3537 & 7.66 & 15,702 & 8.5 & \\
\hline 4 (The lowest) & 7252 & 15.7 & 29,370 & 15.9 & \\
\hline Level of care & & & & & $<0.001$ \\
\hline Hospital center & 18,198 & 39.41 & 60,196 & 32.59 & \\
\hline Regional hospital & 20,537 & 44.47 & 70,919 & 38.39 & \\
\hline Local hospital & 61,045 & 26.44 & 7444 & 16.12 & \\
\hline
\end{tabular}

$p$ : Chi-square/Fisher's exact test on category variables and $t$-test on continue variables. Adjusted HR: multivariable analysis included sex, age, covariates, and comorbidities (hypertension, diabetes mellitus, hyperlipidemia, ischemic heart diseases, congestive heart failure, chronic obstructive pulmonary disease, liver disease, rheumatic disease, connective tissue disease, multiple sclerosis, osteoporosis, Bell's palsy, Parkinson's disease, trachoma, blepharitis, hordeolum, and glaucoma) and medications (listed in Table 1). 


\subsection{Kaplan-Meier Model for the Cumulative Risk of DES}

A Kaplan-Meier graph of the cumulative risks of incident DES is shown in Figure 2, and the log-rank test revealed that the orbital fracture cohort had significantly higher cumulative risks than the control group $(p<0.001)$. The Kaplan-Meier analysis indicated that, in the third year, the incidence of DES was higher in the orbital fracture cohort than in the general population cohort $(p=0.036)$, a finding that persisted until the end of the follow-up (Supplementary Table S3).

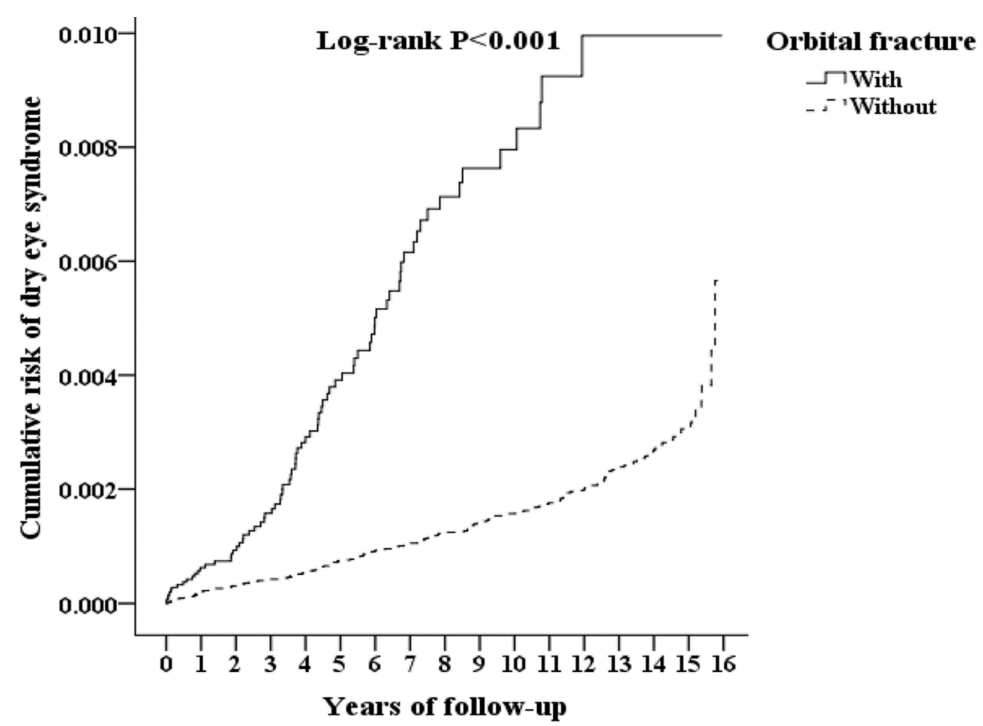

Figure 2. Kaplan-Meier for cumulative risk of dry eye syndrome aged 18 and over stratified by orbital fracture with log-rank test.

\subsection{Comparisons of the Prevalence and Risk of DES}

Orbital fracture patients have a higher risk of DES compared with the control cohort (crude HR = 4.736 (95\% CI, 3.622-6.193); $p<0.001$; Table 3). After adjusting for sex, age, urbanization of residence areas, and other concomitant comorbidities, the adjusted HR was $4.917(95 \% \mathrm{CI}=3.716-6.507 ; p<0.001)$, indicating that patients with orbital fracture had a 4.917-fold increased risk of incident DES compared to controls. Notably, the risk of DES among the female patients with orbital fracture was significantly higher than male patients with orbital fracture, by a multiple of 1.523. Moreover, the adjusted risk of DES in the 18-29-year age group was 3.810-fold than the age group of $\geq 60$ years ( $95 \% \mathrm{CI}=2.367-6.134 ; p<0.001$ ). Of the concomitant comorbidities, MS was the dominant factor for DES, with an adjusted HR of $8.064(95 \% \mathrm{CI}=1.938-33.556 ; p=0.004)$, followed by diffuse diseases of connective tissue (adjusted HR $=6.600(95 \%$ CI, 3.999-10.894); $p<0.001)$ and blepharitis (adjusted HR $=6.246$ (95\% CI, 1.542-25.300); $p=0.010$ ). Furthermore, orbital fracture patients with hordeolum, glaucoma, and Bell's palsy have a greater likelihood of the developing DES. Significant factors of dry eye syndrome include orbital fracture, female, 18- to 29-year age group, MS, diffuse diseases of connective tissue, blepharitis, hordeolum, glaucoma, and Bell's palsy.

\subsection{Hazard Ratios Analysis of DES in the Patients with Orbital Fractures}

Orbital fracture was associated with an increased risk of incident DES regardless of gender, age, season, and level of care (Table 4). The incidence rates of DES in the case group and the control group were 16.63 and 10.37 per $10^{5}$ person-years, respectively. Orbital fracture is the predominant factor among concomitant comorbidities.

\subsection{Hazard Ratio Analysis of DES in the Patients Who Did and Did Not Receive Surgery of Orbital Fracture Subtypes}

The patients with orbital fracture have a higher risk of DES whether they received surgery or not (Table 5). The patients who received surgery had a relatively higher risk of 
developing DES (adjusted HR $=1.097$ [95\% CI, 0.700-1.720]; $p=0.685$ ) compared to patients without surgery. The Kaplan-Meier graph of the cumulative risks of incident DES showed the same result during the 15-year follow-up. Additionally, Table 5 shows the risks of different types of orbital fracture to DES. Patients with orbital roof fracture have a 1.566-fold risk of developing DES compare to orbital floor fracture patients (95\% CI $=0.841-2.915 ; p=0.157$ ).

Table 3. Factors of dry eye syndrome by using Cox regression.

\begin{tabular}{|c|c|c|c|c|c|c|c|c|}
\hline Variables & Crude HR & $95 \% \mathrm{CI}$ & $95 \% \mathrm{CI}$ & $p$ & Adjusted HR & $95 \% \mathrm{CI}$ & $95 \% \mathrm{CI}$ & $p$ \\
\hline Orbital fracture (Reference: without) & 4.736 & 3.622 & 6.193 & $<0.001$ & 4.917 & 3.716 & 6.507 & $<0.001$ \\
\hline Male (Reference: Female) & 1.614 & 1.273 & 2.046 & $<0.001$ & 1.523 & 1.187 & 1.954 & \\
\hline \multicolumn{9}{|l|}{ Age (Reference: $\geq 60$ ) } \\
\hline $18-29$ & 2.332 & 1.539 & 3.533 & $<0.001$ & 3.810 & 2.367 & 6.134 & $<0.001$ \\
\hline $30-39$ & 0.745 & 0.518 & 1.071 & 0.111 & 1.348 & 0.89 & 2.044 & 0.159 \\
\hline $40-49$ & 0.869 & 0.615 & 1.228 & 0.427 & 1.400 & 0.953 & 2.055 & 0.086 \\
\hline $50-59$ & 1.14 & 0.826 & 1.574 & 0.424 & 1.512 & 1.07 & 2.136 & 0.019 \\
\hline \multicolumn{9}{|l|}{ Insured premium (Reference: $<18,000$ ) } \\
\hline $18,000-34,999$ & 0.432 & 0.107 & 1.735 & 0.236 & 0.448 & 0.111 & 1.801 & 0.258 \\
\hline$\geq 35,000$ & 2.755 & 1.027 & 7.392 & 0.044 & 3.375 & 1.252 & 9.101 & 0.016 \\
\hline \multicolumn{9}{|l|}{ Education levels (years) (Reference: <12) } \\
\hline \multicolumn{9}{|l|}{ Comorbidities (Reference: Without) } \\
\hline $\mathrm{DM}$ & 1.354 & 1.059 & 1.730 & 0.016 & 1.243 & 0.936 & 1.650 & 0.133 \\
\hline Hyperlipidemia & 1.619 & 1.223 & 2.144 & 0.001 & 1.472 & 1.08 & 2.006 & 0.014 \\
\hline HTN & 1.350 & 1.068 & 1.705 & 0.012 & 1.080 & 0.801 & 1.456 & 0.613 \\
\hline CKD & 1.003 & 0.718 & 1.401 & 0.988 & 1.007 & 0.514 & 1.358 & 0.098 \\
\hline CAD & 1.448 & 1.132 & 1.853 & 0.003 & 1.269 & 0.943 & 1.708 & 0.115 \\
\hline $\mathrm{CHF}$ & 1.203 & 0.840 & 1.721 & 0.313 & 1.006 & 0.609 & 1.348 & 0.626 \\
\hline Stroke & 1.348 & 1.032 & 1.761 & 0.029 & 1.162 & 0.86 & 1.570 & 0.328 \\
\hline COPD & 1.741 & 1.343 & 2.257 & $<0.001$ & 1.836 & 1.375 & 2.452 & $<0.001$ \\
\hline CLD & 1.126 & 0.854 & 1.486 & 0.399 & 1.172 & 0.875 & 1.570 & 0.288 \\
\hline Osteoporosis & 2.801 & 1.759 & 4.462 & $<0.001$ & 1.993 & 1.211 & 3.280 & 0.007 \\
\hline RA & 5.576 & 3.195 & 9.730 & $<0.001$ & 2.577 & 1.383 & 4.800 & 0.003 \\
\hline CTD & 11.640 & 7.530 & 17.993 & $<0.001$ & 6.600 & 3.999 & 10.894 & $<0.001$ \\
\hline Sarcoidosis & 0.000 & - & - & 0.84 & 0.000 & - & - & 0.979 \\
\hline Trachoma & 0.000 & - & - & 0.577 & 0.000 & - & - & 0.955 \\
\hline MS & 11.569 & 2.879 & 46.492 & 0.001 & 8.064 & 1.938 & 33.556 & 0.004 \\
\hline Bell's palsy & 3.719 & 1.756 & 7.874 & 0.001 & 2.192 & 1.028 & 4.674 & 0.042 \\
\hline Parkinson disease & 1.959 & 1.145 & 3.353 & 0.014 & 1.778 & 1.026 & 3.081 & 0.04 \\
\hline Blepharitis & 7.655 & 1.905 & 30.763 & 0.004 & 6.246 & 1.542 & 25.300 & 0.01 \\
\hline Hordeolum & 8.100 & 3.018 & 21.740 & $<0.001$ & 5.734 & 2.055 & 16.003 & 0.001 \\
\hline Glaucoma & 6.067 & 3.808 & 9.666 & $<0.001$ & 4.960 & 3.071 & 8.009 & $<0.001$ \\
\hline CCI_R & 1.008 & 0.838 & 1.142 & 0.782 & 1.050 & 0.912 & 1.208 & 0.496 \\
\hline \multicolumn{9}{|l|}{ Medications (Reference: Without) } \\
\hline Anti-HTN drugs & 1.453 & 0.88 & 1.979 & 0.546 & 1.210 & 0.797 & 1.871 & 0.514 \\
\hline Antidepressants drugs & 1.103 & 0.794 & 1.29 & 0.464 & 1.092 & 0.722 & 1.238 & 0.478 \\
\hline Anti-CA drugs & 0.981 & 0.357 & 1.599 & 0.785 & 0.946 & 0.332 & 1.524 & 0.762 \\
\hline Anti-Parkinson drugs & 1.584 & 0.877 & 2.601 & 0.578 & 1.423 & 0.758 & 2.34 & 0.588 \\
\hline Anti-ulcer drugs & & 0.300 & 2.990 & 0.876 & 1.678 & 0.245 & 2.593 & 0.835 \\
\hline Muscle spasm drugs & 1.121 & 0.599 & 1.867 & 0.351 & 1.104 & 0.532 & 1.82 & 0.333 \\
\hline Decongestant drugs & 1.266 & 0.451 & 2.384 & 0.623 & 1.298 & 0.489 & 2.415 & 0.601 \\
\hline Antihistamines drugs & 1.986 & 0.230 & 4.350 & 0.927 & 1.834 & 0.202 & 4.030 & 0.911 \\
\hline Anesthetics drugs & 0.989 & 0.149 & 2.846 & 0.933 & 1.006 & 0.164 & 3.000 & 0.976 \\
\hline \multicolumn{9}{|l|}{ Season (Reference: Spring) } \\
\hline Summer & 0.781 & 0.566 & 1.079 & 0.134 & 0.752 & 0.544 & 1.039 & 0.084 \\
\hline Autumn & 0.686 & 0.495 & 0.951 & 0.024 & 0.641 & 0.462 & 0.890 & 0.008 \\
\hline Winter & 0.841 & 0.609 & 1.163 & 0.296 & 0.828 & 0.599 & 1.145 & 0.254 \\
\hline \multicolumn{9}{|l|}{ Location (Reference: Northern Taiwan) } \\
\hline Middle Taiwan & 0.671 & 0.494 & 0.913 & 0.011 & \multicolumn{4}{|c|}{ Multicollinearity with urbanization level } \\
\hline Southern Taiwan & 0.890 & 0.667 & 1.188 & 0.429 & \multicolumn{4}{|c|}{ Multicollinearity with urbanization level } \\
\hline Eastern Taiwan & 1.324 & 0.882 & 1.987 & 0.176 & \multicolumn{4}{|c|}{ Multicollinearity with urbanization level } \\
\hline Outlets islands & 0 & - & - & 0.912 & \multicolumn{4}{|c|}{ Multicollinearity with urbanization level } \\
\hline \multicolumn{9}{|l|}{ Urbanization level (Reference: 4) } \\
\hline 1 (The highest) & 1.564 & 1.088 & 2.248 & 0.016 & 1.181 & 0.801 & 1.740 & 0.401 \\
\hline 2 & 1.501 & 1.024 & 2.202 & 0.037 & 1.026 & 0.587 & 1.796 & 0.927 \\
\hline 3 & 1.071 & 0.616 & 1.863 & 0.807 & 1.011 & 0.655 & 1.560 & 0.960 \\
\hline \multicolumn{9}{|l|}{ Level of care (Reference: Local hospital) } \\
\hline Hospital center & 2.537 & 1.753 & 3.671 & $<0.001$ & 2.776 & 1.830 & 4.213 & $<0.001$ \\
\hline Regional hospital & 1.646 & 1.134 & 2.390 & 0.009 & 1.723 & 1.178 & 2.521 & 0.005 \\
\hline
\end{tabular}

HR, hazard ratio; CI, confidence interval; Adjusted HR, Adjusted variables listed in the table. Adjusted HR: multivariable analysis included sex, age, covariates, and comorbidities (hypertension, diabetes mellitus, hyperlipidemia, ischemic heart diseases, congestive heart failure, chronic obstructive pulmonary disease, liver disease, rheumatic disease, connective tissue disease, multiple sclerosis, osteoporosis, Bell's palsy, Parkinson's disease, trachoma, blepharitis, hordeolum, and glaucoma) and medications (listed in Table 1). 
Table 4. Factors of dry eye syndrome stratified by variables listed in the table using Cox regression.

\begin{tabular}{|c|c|c|c|c|c|c|c|c|c|c|}
\hline \multirow{2}{*}{$\begin{array}{l}\text { Orbital Fracture } \\
\text { Stratified }\end{array}$} & \multicolumn{3}{|c|}{ With } & \multicolumn{3}{|c|}{ Without (Reference) } & \multicolumn{4}{|c|}{ With vs. Without (Reference) } \\
\hline & Events & PYs & Rate (per $10^{5} \mathrm{PYs}$ ) & Events & PYs & Rate (per $10^{5} \mathrm{PYs}$ ) & Adjusted HR & $95 \% \mathrm{CI}$ & $95 \% \mathrm{CI}$ & $p$ \\
\hline Total & 79 & $475,088.93$ & 16.63 & 206 & $1,985,645.35$ & 10.37 & 4.917 & 3.716 & 6.507 & $<0.001$ \\
\hline \multicolumn{11}{|l|}{ Gender } \\
\hline Female & 35 & $139,432.71$ & 25.10 & 88 & $576,346.32$ & 15.27 & 5.043 & 3.811 & 6.674 & $<0.001$ \\
\hline \multicolumn{11}{|l|}{ Age (yrs) } \\
\hline $18-29$ & 19 & $55,747.48$ & 34.08 & 16 & $90,383.93$ & 17.70 & 5.906 & 4.463 & 7.816 & $<0.001$ \\
\hline 30-39 & 11 & $92,878.70$ & 11.84 & 28 & $376,713.68$ & 7.43 & 4.888 & 3.694 & 6.468 & $<0.001$ \\
\hline $40-49$ & 11 & $79,470.94$ & 13.84 & 30 & $355,420.38$ & 8.44 & 5.031 & 3.802 & 6.657 & $<0.001$ \\
\hline$\geq 60$ & 20 & $166,088.82$ & 12.04 & 92 & $832,445.53$ & 11.05 & 3.343 & 2.526 & 4.423 & $<0.001$ \\
\hline \multicolumn{11}{|l|}{ Insured premium (NT\$) } \\
\hline$<18,000$ & 77 & $465,576.68$ & 16.54 & 202 & $1,943,410.70$ & 10.39 & 4.881 & 3.689 & 6.459 & $<0.001$ \\
\hline $18,000-34,999$ & 0 & 7798.00 & 0.00 & 2 & $31,835.37$ & 6.28 & 0.000 & - & - & 0.979 \\
\hline$\geq 35,000$ & 2 & 1714.24 & 116.67 & 2 & $10,399.29$ & 19.23 & 18.610 & 14.064 & 24.627 & $<0.001$ \\
\hline \multicolumn{11}{|l|}{ Education levels (yrs) } \\
\hline$<12$ & 46 & $248,072.48$ & 18.54 & 138 & $1,045,925.08$ & 13.19 & 4.311 & 3.258 & 5.705 & $<0.001$ \\
\hline$\geq 12$ & 33 & $227,016.45$ & 14.54 & 68 & $939,720.27$ & 7.24 & 6.163 & 4.657 & 8.155 & $<0.001$ \\
\hline \multicolumn{11}{|l|}{$\mathrm{DM}$} \\
\hline Without & 49 & $362,152.29$ & 13.53 & 128 & $1,434,066.93$ & 8.93 & 4.650 & 3.514 & 6.154 & $<0.001$ \\
\hline Without & 62 & $431,204.03$ & 14.38 & 152 & $1,687,032.31$ & 9.01 & 4.896 & 3.700 & 6.478 & $<0.001$ \\
\hline With & 17 & $43,884.89$ & 38.74 & 54 & $298,613.04$ & 18.08 & 6.572 & 4.966 & 8.696 & $<0.001$ \\
\hline \multicolumn{11}{|l|}{ HTN } \\
\hline Without & 54 & $305,101.23$ & 17.70 & 129 & $1,147,122.95$ & 11.25 & 4.828 & 3.649 & 6.389 & $<0.001$ \\
\hline With & 25 & $169,987.70$ & 14.71 & 77 & $838,522.40$ & 9.18 & 4.913 & 3.713 & 6.502 & $<0.001$ \\
\hline \multicolumn{11}{|l|}{ CKD } \\
\hline Without & 69 & $433,018.39$ & 15.93 & 172 & $1,700,934.97$ & 10.11 & 4.834 & 3.653 & 6.397 & $<0.001$ \\
\hline With & 10 & $42,070.54$ & 23.77 & 34 & $284,710.38$ & 11.94 & 6.106 & 4.614 & 8.080 & $<0.001$ \\
\hline \multicolumn{11}{|l|}{ CAD } \\
\hline Without & 59 & $391,342.67$ & 15.08 & 137 & $1,463,436.21$ & 9.36 & 4.940 & 3.733 & 6.538 & $<0.001$ \\
\hline With & 20 & $83,746.26$ & 23.88 & 69 & $522,209.14$ & 13.21 & 5.545 & 4.190 & 7.337 & $<0.001$ \\
\hline \multicolumn{11}{|l|}{$\mathrm{CHF}$} \\
\hline Without & 71 & $444,230.54$ & 15.98 & 177 & $1,781,171.96$ & 9.94 & 4.934 & 3.729 & 6.529 & $<0.001$ \\
\hline With & 8 & $30,858.39$ & 25.92 & 29 & $204,473.39$ & 14.18 & 5.608 & 4.238 & 7.420 & $<0.001$ \\
\hline \multicolumn{11}{|l|}{ Stroke } \\
\hline Without & 57 & $381,249.02$ & 14.95 & 150 & $1,591,558.41$ & 9.42 & 4.866 & 3.678 & 6.440 & $<0.001$ \\
\hline With & 22 & $93,839.91$ & 23.44 & 56 & $394,086.94$ & 14.21 & 5.061 & 3.825 & 6.697 & $<0.001$ \\
\hline
\end{tabular}


Table 4. Cont.

\begin{tabular}{|c|c|c|c|c|c|c|c|c|c|c|}
\hline \multirow{2}{*}{$\begin{array}{l}\text { Orbital Fracture } \\
\text { Stratified }\end{array}$} & \multicolumn{3}{|c|}{ With } & \multicolumn{3}{|c|}{ Without (Reference) } & \multicolumn{4}{|c|}{ With vs. Without (Reference) } \\
\hline & Events & PYs & Rate (per $10^{5} \mathrm{PYs}$ ) & Events & PYs & Rate (per $10^{5} \mathrm{PYs}$ ) & Adjusted HR & $95 \% \mathrm{CI}$ & $95 \% \mathrm{CI}$ & $p$ \\
\hline \multicolumn{11}{|l|}{ COPD } \\
\hline Without & 59 & $410,992.47$ & 14.36 & 142 & $1,623,732.70$ & 8.75 & 5.036 & 3.805 & 6.664 & $<0.001$ \\
\hline \multicolumn{11}{|l|}{ CLD } \\
\hline Without & 59 & $404,963.89$ & 14.57 & 153 & $1,568,057.99$ & 9.76 & 4.581 & 3.462 & 6.061 & $<0.001$ \\
\hline With & 20 & $70,125.04$ & 28.52 & 53 & $417,587.35$ & 12.69 & 6.894 & 5.209 & 9.122 & $<0.001$ \\
\hline \multicolumn{11}{|l|}{ Osteoporosis } \\
\hline Without & 73 & $468,938.39$ & 15.57 & 188 & $1,934,814.70$ & 9.72 & 4.915 & 3.714 & 6.504 & $<0.001$ \\
\hline \multicolumn{11}{|l|}{ RA } \\
\hline Without & 77 & $472,765.82$ & 16.29 & 198 & $1,968,579.60$ & 10.06 & 4.968 & 3.754 & 6.574 & $<0.001$ \\
\hline With & 2 & 2323.11 & 86.09 & 8 & $17,065.75$ & 46.88 & 5.634 & 4.258 & 7.455 & $<0.001$ \\
\hline \multicolumn{11}{|l|}{ CTD } \\
\hline Without & 73 & $472,713.12$ & 15.44 & 191 & $1,971,480.28$ & 9.69 & 4.890 & 3.695 & 6.471 & $<0.001$ \\
\hline With & 6 & 2375.81 & 252.55 & 15 & $14,165.06$ & 105.89 & 7.316 & 5.529 & 9.681 & $<0.001$ \\
\hline \multicolumn{11}{|l|}{ Sarcoidosis } \\
\hline Without & 79 & $474,923.46$ & 16.63 & 204 & $1,985,048.07$ & 10.28 & 4.965 & 3.752 & 6.571 & $<0.001$ \\
\hline With & 0 & 165.47 & 0.00 & 2 & 597.28 & 334.85 & 0.000 & - & - & 0.976 \\
\hline \multicolumn{11}{|l|}{ Trachoma } \\
\hline \multicolumn{11}{|l|}{ MS } \\
\hline Without & 78 & $474,990.41$ & 16.42 & 205 & $1,984,386.50$ & 10.33 & 4.876 & 3.685 & 6.453 & $<0.001$ \\
\hline With & 1 & 98.52 & 1015.01 & 1 & 1258.85 & 79.44 & 39.198 & 29.622 & 51.870 & $<0.001$ \\
\hline \multicolumn{11}{|l|}{ Bell's palsy } \\
\hline Without & 75 & $462,955.91$ & 16.20 & 204 & $1,975,921.80$ & 10.32 & 4.814 & 3.638 & 6.370 & $<0.001$ \\
\hline With & 4 & $12,133.02$ & 32.97 & 2 & 9723.55 & 20.57 & 4.917 & 3.716 & 6.507 & $<0.001$ \\
\hline \multicolumn{11}{|l|}{ Parkinson disease } \\
\hline Without & 76 & $464,221.80$ & 16.37 & 199 & $1,935,404.09$ & 10.28 & 4.885 & 3.691 & 6.464 & $<0.001$ \\
\hline With & 3 & $10,867.13$ & 27.61 & 7 & $50,241.26$ & 13.93 & 6.078 & 4.593 & 8.043 & $<0.001$ \\
\hline \multicolumn{11}{|l|}{ Blepharitis } \\
\hline Without & 76 & $474,816.42$ & 16.01 & 204 & $1,983,792.45$ & 10.28 & 4.775 & 3.608 & 6.319 & $<0.001$ \\
\hline With & 3 & 272.51 & 1100.88 & 2 & 1852.90 & 107.94 & 31.288 & 23.644 & 41.403 & $<0.001$ \\
\hline \multicolumn{11}{|l|}{ Hordeolum } \\
\hline Without & 76 & $474,256.27$ & 16.03 & 202 & $1,982,298.80$ & 10.19 & 4.824 & 3.646 & 6.384 & $<0.001$ \\
\hline With & 3 & 832.66 & 360.29 & 4 & 3346.55 & 119.53 & 9.247 & 6.988 & 12.237 & $<0.001$ \\
\hline
\end{tabular}


Table 4. Cont.

\begin{tabular}{|c|c|c|c|c|c|c|c|c|c|c|}
\hline \multirow{2}{*}{$\begin{array}{l}\text { Orbital Fracture } \\
\text { Stratified }\end{array}$} & \multicolumn{3}{|c|}{ With } & \multicolumn{3}{|c|}{ Without (Reference) } & \multicolumn{4}{|c|}{ With vs. Without (Reference) } \\
\hline & Events & PYs & Rate (per $10^{5} \mathrm{PYs}$ ) & Events & PYs & Rate (per $10^{5} \mathrm{PYs}$ ) & Adjusted HR & $95 \% \mathrm{CI}$ & $95 \% \mathrm{CI}$ & $p$ \\
\hline \multicolumn{11}{|l|}{ Glaucoma } \\
\hline Without & 76 & $470,302.16$ & 16.16 & 198 & $1,962,759.09$ & 10.09 & 4.914 & 3.714 & 6.503 & $<0.001$ \\
\hline \multicolumn{11}{|l|}{ Antidepressants drugs } \\
\hline \multicolumn{11}{|l|}{ Anti-HTN drugs } \\
\hline Without & 56 & $393,561.26$ & 14.23 & 139 & $1,541,068.35$ & 9.02 & 4.840 & 3.657 & 6.404 & $<0.001$ \\
\hline With & 23 & $81,527.67$ & 28.21 & 67 & $444,577.00$ & 15.07 & 5.743 & 4.340 & 7.599 & $<0.001$ \\
\hline Without & 50 & $447,758.69$ & 11.17 & 144 & $1,880,811.35$ & 7.66 & 4.474 & 3.381 & 5.921 & $<0.001$ \\
\hline \multicolumn{11}{|l|}{ Anti-CA drugs } \\
\hline Without & 66 & $473,689.49$ & 13.93 & 166 & $1,979,679.10$ & 8.39 & 5.097 & 3.852 & 6.745 & $<0.001$ \\
\hline With & 13 & 1399.44 & 928.94 & 40 & 5966.25 & 670.44 & 4.251 & 3.212 & 5.625 & $<0.001$ \\
\hline \multicolumn{11}{|l|}{ Anti-Parkinson drugs } \\
\hline Without & 53 & $471,981.35$ & 11.23 & 127 & $1,965,059.10$ & 6.46 & 5.330 & 4.028 & 7.053 & $<0.001$ \\
\hline With & 26 & 3107.58 & 836.66 & 79 & $20,586.25$ & 383.75 & 6.688 & 5.054 & 8.851 & $<0.001$ \\
\hline \multicolumn{11}{|l|}{ Anti-ulcer drugs } \\
\hline Without & 61 & $473,740.94$ & 12.88 & 187 & $1,983,194.35$ & 9.43 & 4.189 & 3.166 & 5.544 & $<0.001$ \\
\hline With & 18 & 1347.99 & 1335.32 & 19 & 2451.00 & 775.19 & 5.284 & 3.993 & 6.993 & $<0.001$ \\
\hline \multicolumn{11}{|l|}{ Muscle spasm drugs } \\
\hline \multicolumn{11}{|l|}{ Decongestant drugs } \\
\hline Without & 61 & $471,086.12$ & 12.95 & 171 & $1,971,509.10$ & 8.67 & 4.580 & 3.461 & 6.060 & $<0.001$ \\
\hline With & 18 & 4002.81 & 449.68 & 35 & $14,136.25$ & 247.59 & 5.572 & 4.211 & 7.373 & $<0.001$ \\
\hline \multicolumn{11}{|l|}{ Antihistamines drugs } \\
\hline Without & 65 & $463,399.49$ & 14.03 & 173 & $1,937,281.10$ & 8.93 & 4.819 & 3.641 & 6.376 & $<0.001$ \\
\hline With & 14 & $11,689.44$ & 119.77 & 33 & $48,364.25$ & 68.23 & 5.385 & 4.069 & 7.126 & $<0.001$ \\
\hline \multicolumn{11}{|l|}{ Anesthetics drugs } \\
\hline Without & 59 & $473,885.00$ & 12.45 & 164 & $1,981,022.85$ & 8.28 & 4.614 & 3.486 & 6.105 & $<0.001$ \\
\hline With & 20 & 1203.93 & 1661.23 & 42 & 4622.50 & 908.60 & 5.609 & 4.239 & 7.422 & $<0.001$ \\
\hline \multicolumn{11}{|l|}{ Season } \\
\hline Spring & 25 & $105,038.76$ & 23.80 & 56 & $462,940.42$ & 12.10 & 6.036 & 4.561 & 7.987 & $<0.001$ \\
\hline Summer & 18 & $121,136.73$ & 14.86 & 51 & $508,305.36$ & 10.03 & 4.543 & 3.433 & 6.012 & $<0.001$ \\
\hline Autumn & 13 & $137,573.52$ & 9.45 & 48 & $548,918.18$ & 8.74 & 3.315 & 2.505 & 4.387 & $<0.001$ \\
\hline Winter & 23 & $111,339.92$ & 20.66 & 51 & $465,481.39$ & 10.96 & 5.784 & 4.371 & 7.654 & $<0.001$ \\
\hline
\end{tabular}


Table 4. Cont.

\begin{tabular}{|c|c|c|c|c|c|c|c|c|c|c|}
\hline \multirow{2}{*}{$\begin{array}{l}\text { Orbital Fracture } \\
\text { Stratified }\end{array}$} & \multicolumn{3}{|c|}{ With } & \multicolumn{3}{|c|}{ Without (Reference) } & \multicolumn{4}{|c|}{ With vs. Without (Reference) } \\
\hline & Events & PYs & Rate (per $10^{5} \mathrm{PYs}$ ) & Events & PYs & Rate (per $10^{5}$ PYs) & Adjusted HR & $95 \%$ CI & $95 \% \mathrm{CI}$ & $p$ \\
\hline \multicolumn{11}{|l|}{ Urbanization level } \\
\hline 1 (The highest) & 29 & $135,162.15$ & 21.46 & 55 & $589,023.47$ & 9.34 & 7.049 & 5.327 & 9.328 & $<0.001$ \\
\hline 3 & 15 & $44,460.22$ & 33.74 & 44 & $171,456.22$ & 25.66 & 4.033 & 3.048 & 5.337 & $<0.001$ \\
\hline 4 (The lowest) & 14 & $92,313.67$ & 15.17 & 54 & $356,642.70$ & 15.14 & 3.073 & 2.322 & 4.066 & $<0.001$ \\
\hline \multicolumn{11}{|l|}{ Level of care } \\
\hline Hospital center & 35 & $140,015.22$ & 25.00 & 80 & $637,196.38$ & 12.55 & 6.108 & 4.616 & 8.083 & $<0.001$ \\
\hline Regional hospital & 29 & $224,241.18$ & 12.93 & 75 & $899,300.83$ & 8.34 & 4.757 & 3.595 & 6.295 & $<0.001$ \\
\hline
\end{tabular}

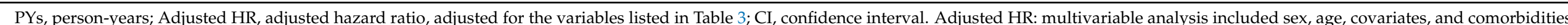

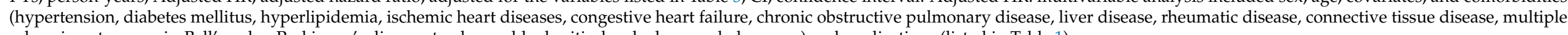
sclerosis, osteoporosis, Bell's palsy, Parkinson's disease, trachoma, blepharitis, hordeolum, and glaucoma) and medications (listed in Table 1).

Table 5. Factors of dry eye syndrome with/without surgery of orbital fracture subtypes using Cox regression.

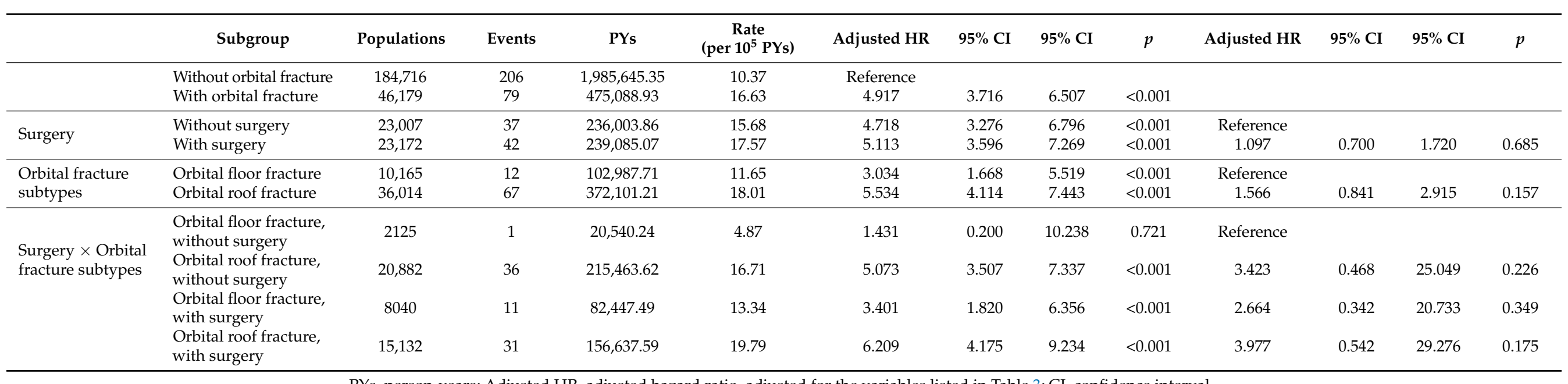

PYs, person-years; Adjusted HR, adjusted hazard ratio, adjusted for the variables listed in Table 3; CI, confidence interval. 


\section{Discussion}

The association between orbital fracture and DES remains unclear. Only one previous study pointed out a slightly lower amount of tear film in the affected eye compared with the unaffected eye among orbital floor fracture patients [11]. However, the study result did not meet the diagnosis criteria of DES. The study included only 23 participants, and the tear film quantity was measured by phenol red thread test. Furthermore, 10 of 23 patients $(43 \%)$ had subnormal tear film values. Eight patients revealed a decreased tear production, while two displayed an excess in tear production (epiphora). Additionally, there was a considerably weak relationship between phenol red thread tests and symptoms of dry eyes [19]. Thus, a population-based research with large study number, confirmed diagnosis of DES, and multiple potential risk factors is warranted.

Using a population-based dataset, our study demonstrated that orbital fracture and DES were significantly associated even after adjusting for the patients' demographic characteristics, comorbidities, and clinical pertinent covariates. During a 15-year follow-up, patients in the case group had an increased risk of DES, with an overall adjusted HR of 4.917 (95\% CI, 3.716-6.507; $p<0.001$ ), which was a 391.7\% increase in the risk of developing DES compared to the controls. Notably, orbital roof fracture patients had relatively higher risk of developing DES compared to orbital floor fracture patients (adjusted HR $=1.566$ $(95 \% \mathrm{CI}=0.841-2.915) ; p=0.157)$.

The possible mechanisms between the orbital fracture and DES are complex and may be associated with variable multi-factors. One plausible explanation for our observation was that the rate of DES occurrence following the orbital fracture was attributed to lacrimal gland injury. The lacrimal gland is located anteriorly in the superolateral aspect of the orbit, within the lacrimal fossa. The major source of tear fluid is from the lacrimal gland [20]. This suggests that the anatomical disruption and mechanical compression from orbital trauma contributed to the resulting injury of the lacrimal gland, including hematoma, edema, and vascular insufficiency. A previous study involved 200 cases of closed head injury admitted to a major teaching hospital. Ocular involvement was found in 167 (83.5\%) cases, and two cases (1\%) had lacrimal gland prolapse [21].

The inflammatory process during orbital fracture may activate the inflammatory reaction in the dry eye developing cycle. DES results from a combination of factors. One diagnostic classification scheme divides dry eye patients into those with aqueous tear deficiency and those with evaporative dry eye [22]. In aqueous tear deficiency, T-cellmediated inflammation of the lacrimal gland occurs, which results in diminished secretion of the aqueous layer of the tear film and the propagation of inflammatory mediators on the ocular surface $[23,24]$. Therefore, severe orbital fracture, especially orbital roof fracture, may contribute to the development of DES, as demonstrated in the current study.

Isolated orbital roof fractures are uncommon. However, it is estimated that $1-9 \%$ of facial bone fractures involve the orbital roof $[25,26]$. In addition, orbital roof fractures are frequently associated with high-energy injuries to the head and face [26,27]. Several of these patients (13-19\%) have multi-system injuries, most of which are neurologic (57-90\%) [27-29]. These neurologic deficits could lead to higher risk of DES among orbital roof fracture patients.

In this study, we further evaluated the risks of DES associated with each comorbidity and studied the impact of orbital fracture on DES in association with these comorbidities (Tables 3 and 4). Our results are consistent with those of previous epidemiological studies, which have revealed that female patients and several diseases are prone to DES, including osteoporosis, arthritis, connective tissue disease, and hyperlipidemia $[15,16,30]$. However, the tendency of DES increasing with age was not significant in our study. In contrast, orbital fracture patients aged 18-29 years had higher risks of DES than those aged $\geq 30$ years. This might be due to the higher prevalence of orbital fracture in our cohort of patients in their thirties. Nevertheless, this does not neglect the fact that orbital fractures play a significant role in DES among the younger population. 
A high prevalence of DES among patients with glaucoma and MS was observed in previous studies [31-35]. Our study further confirms the increase risk of DES among patients with glaucoma and MS. The results suggest that preventive therapy and current therapeutic efficacy should be considered.

However, the results should be interpreted within the context of the following limitations. First, although we had done our best to adjust for the influence of socioeconomic status, there were several confounding factors for orbital fracture and DES that we could not obtain from the NHIRD, such as contact lens use, smoking habits, caffeine use, alcohol consumption, nutrition, and video display terminal exposure, including the use of mobile and electronic devices. Second, patients diagnosed with orbital fracture and incident DES were identified based on the insurance claims data rather than real medical documents. The severity and laterality of the injuries were not available because of the lack of detailed clinical information in the ICD-9 coding system. Third, the retrospective nature of study design may reduce the homogeneity of the patient population, even after propensity score matching with multiple systemic diseases. Finally, we did not analyze the type of orbital fracture (i.e., fracture of medial wall of orbit, naso-orbital ethmoid bone, or lateral wall of the orbit) due to the limitation of the ICD-9 coding system. Despite these limitations, the strength of this study is that it reports longitudinal results on the association between orbital fracture and the risk of subsequent DES events in a nationwide, population-based cohort.

\section{Conclusions}

Our study clarified the relationship between orbital fracture and increased risk of developing subsequent DES. Early recognition by thorough examinations with increased awareness in the clinical setting could preserve visual function, eliminate ocular symptoms, and prevent further complications.

Supplementary Materials: The following are available online at https: / www.mdpi.com/article/ 10.3390/healthcare9050605/s1, Table S1: Years of follow-up. Table S2: Years to dry eye syndrome. Table S3: The incidence of DES in the orbital fracture group and in the general population group.

Author Contributions: Conceptualization, C.Y.-Y.H. and H.-T.L.; methodology, J.C.-Y.T.; software, C.H.C.; validation, C.-A.S. and W.-C.C.; formal analysis, C.-H.C.; resources, C.-H.C.; data curation, J.C.Y.T.; writing—original draft preparation, C.Y.-Y.H.; writing—review and editing, H.-T.L.; supervision, H.-T.L.; project administration, W.-C.C.; funding acquisition, W.-C.C. All authors have read and agreed to the published version of the manuscript.

Funding: This study was supported by the Tri-Service General Hospital Research Foundation (TSGHB-110012); the sponsor had no role in the study design, data collection and analysis, decision to publish, or preparation of the manuscript.

Institutional Review Board Statement: The study was conducted according to the guidelines of the Declaration of Helsinki, and approved by the Institutional Review Board of Tri-Service General Hospital, National Defense Medical Center (TSGHIRB No.: B-110-02).

Acknowledgments: We appreciate the Health and Welfare Data Science Center, Ministry of Health and Welfare (HWDC, MOHW), Taiwan, for providing the National Health Insurance Research Database (NHIRD).

Conflicts of Interest: The authors declare no conflict of interest.

\section{References}

1. Joseph, J.M.; Glavas, I.P. Orbital fractures: A review. Clin. Ophthalmol. 2011, 5, 95-100. [CrossRef]

2. Bullock, J.D.; Warwar, R.E.; Ballal, D.R.; Ballal, R.D. Mechanisms of orbital floor fractures: A clinical, experimental, and theoretical study. Trans. Am. Ophthalmol. Soc. 1999, 97, 87-113.

3. Koenen, L.; Waseem, M. Orbital Floor (Blowout) Fracture. In Stat; Pearls Publishing: Treasure Island, FL, USA, 2019.

4. Bord, S.P.; Linden, J. Trauma to the Globe and Orbit. Emerg. Med. Clin. N. Am. 2008, 26, 97-123. [CrossRef]

5. Gosau, M.; Schöneich, M.; Draenert, F.G.; Ettl, T.; Driemel, O.; Reichert, T.E. Retrospective analysis of orbital floor fracturesComplications, outcome and review of literature. Clin. Oral Investig. 2011, 15, 305-313. [CrossRef] 
6. Hwang, K.; You, S.H.; Sohn, I.A. Analysis of orbital bone fractures: A 12-year study of 391 patients. J. Craniofac. Surg. 2009, 20, 1218-1223. [CrossRef]

7. Shere, J.L.; Boole, J.R.; Holtel, M.R.; Amoroso, P.J. An Analysis of 3599 Midfacial and 1141 Orbital Blowout Fractures Among 4426 United States Army Soldiers, 1980-2000. Otolaryngol. Neck Surg. 2004, 130, 164-170. [CrossRef]

8. Chiang, E.; Saadat, L.V.; Spitz, J.A.; Bryar, P.J.; Chambers, C.B. Etiology of orbital fractures at a level I trauma center in a large metropolitan city. Taiwan J. Ophthalmol. 2016, 6, 26-31. [CrossRef] [PubMed]

9. Cruz, A.A.V.; Eichenberger, G.C.D. Epidemiology and management of orbital fractures. Curr. Opin. Ophthalmol. 2004, 15, 416-421. [CrossRef] [PubMed]

10. Kim, Y.S.; Kim, J.H.; Hwang, K. The Frequency of Decreased Visual Acuity in Orbital Fractures. J. Craniofac. Surg. 2015, 26, 1581-1583. [CrossRef]

11. Pansell, T.; Alinasab, B.; Westermark, A.; Beckman, M.; Abdi, S. Ophthalmologic Findings in Patients with Non-Surgically Treated Blowout Fractures. Craniomaxillofac. Trauma Reconstr. 2012, 5, 1-5. [CrossRef] [PubMed]

12. Goto, E.; Yagi, Y.; Matsumoto, Y.; Tsubota, K. Impaired functional visual acuity of dry eye patients. Am. J. Ophthalmol. 2002, 133, 181-186. [CrossRef]

13. Tutt, R.; Bradley, A.; Begley, C.; Thibos, L.N. Optical and visual impact of tear break-up in human eyes. Investig. Ophthalmol. Vis. Sci. 2000, 41, 4117-4123. [PubMed]

14. Koh, S. Mechanisms of Visual Disturbance in Dry Eye. Cornea 2016, 35, S83-S88. [CrossRef] [PubMed]

15. Moss, S.E.; Klein, R.; Klein, B.E.K. Prevalence of and Risk Factors for Dry Eye Syndrome. Arch. Ophthalmol. 2000, 118, 1264-1268. [CrossRef] [PubMed]

16. Yang, W.J.; Yang, Y.N.; Cao, J.; Man, Z.H.; Yuan, J.; Xiao, X.; Xing, Y.Q. Risk factors for dry eye syndrome: A retrospective case-control study. Optom. Vis. Sci. 2015, 92, e199-e205. [CrossRef] [PubMed]

17. Cheng, T.-M. Taiwan's National Health Insurance System: High Value for the Dollar, Six Countries, Six Reform Models: The Health Reform Experience of ISRAEL, The Netherlands, New Zealand, Singapore, Switzerland and Taiwan; World Scientific: Hackensack, NJ, USA, 2009; pp. 171-204.

18. Cheng, C.-L.; Kao, Y.-H.Y.; Lin, S.-J.; Lee, C.-H.; Lai, M.L. Validation of the national health insurance research database with ischemic stroke cases in Taiwan. Pharmacoepidemiol. Drug Saf. 2010, 20, 236-242. [CrossRef]

19. A Saleh, T.; McDermott, B.; Bates, A.K.; Ewings, P. Phenol red thread test vs Schirmer's test: A comparative study. Eye 2005, 20, 913-915. [CrossRef]

20. Obata, H. Anatomy and Histopathology of the Human Lacrimal Gland. Cornea 2006, 25, S82-S89. [CrossRef]

21. Kulkarni, A.R.; Aggarwal, S.P.; Deshpande, M.D.; Walimbe, P.B.; Labhsetwar, A.S. Ocular manifestations of head injury: A clinical study. Eye 2004, 19, 1257-1263. [CrossRef]

22. Coroneo, M.T. High and dry: An update on dry eye syndrome. Ophthalmol. Clin. 2013, 14, 53-61.

23. Zoukhri, D. Mechanisms Involved in Injury and Repair of the Murine lacrimal Gland: Role of Programmed Cell Death and Mesenchymal Stem Cells. Ocul. Surf. 2010, 8, 60-69. [CrossRef]

24. Jain, S.M.; Bhavsar, A.S.; Bhavsar, S.G. A review on recent advances in dry eye: Pathogenesis and management. Oman J. Ophthalmol. 2011, 4, 50-56. [CrossRef] [PubMed]

25. Penfold, C.; Lang, D.; Evans, B. The management of orbital roof fractures. Br. J. Oral Maxillofac. Surg. 1992, 30, 97-103. [CrossRef]

26. Haug, R.H. Management of the trochlea of the superior oblique muscle in the repair of orbital roof trauma. J. Oral Maxillofac. Surg. 2000, 58, 602-606. [CrossRef]

27. Kim, J.W.; Bae, T.H.; Kim, W.S.; Kim, H.K. Early Reconstruction of Orbital Roof Fractures: Clinical Features and Treatment Outcomes. Arch. Plast. Surg. 2012, 39, 31-35. [CrossRef]

28. Donahue, D.J.; Smith, K.; Church, E.; Chadduck, W.M. Intracranial neurological injuries associated with orbital fracture. Pediatr. Neurosurg. 1997, 26, 261-268. [CrossRef] [PubMed]

29. Flanagan, J.C.; McLachlan, D.L.; Shannon, G.M. Orbital roof fractures: Neurologic and neurosurgical considerations. Ophthalmology 1980, 87, 325-329. [CrossRef]

30. Jeng, Y.T.; Lin, S.Y.; Hu, H.Y.; Lee, O.K.; Kuo, L.L. Osteoporosis and dry eye syndrome: A previously unappreciated association that may alert active prevention of fall. PLoS ONE 2018, 13, e0207008. [CrossRef]

31. Ali, F.S.; Akpek, E.K. Glaucoma and Dry Eye. Ophthalmology 2009, 116, 1232. [CrossRef]

32. Leung, E.W.; Medeiros, F.A.; Weinreb, R.N. Prevalence of Ocular Surface Disease in Glaucoma Patients. J. Glaucoma 2008, 17, 350-355. [CrossRef]

33. Servat, J.J.; Bernardino, C.R. Effects of Common Topical Antiglaucoma Medications on the Ocular Surface, Eyelids and Periorbital Tissue. Drugs Aging 2011, 28, 267-282. [CrossRef] [PubMed]

34. Fechtner, R.D.; Godfrey, D.G.; Budenz, D.; A Stewart, J.; Stewart, W.C.; Jasek, M.C. Prevalence of Ocular Surface Complaints in Patients with Glaucoma Using Topical Intraocular Pressure-Lowering Medications. Cornea 2010, 29, 618-621. [CrossRef] [PubMed]

35. De Seze, J.; Devos, D.; Castelnovo, G.; Labauge, P.; Dubucquoi, S.; Stojkovic, T.; Ferriby, D.; Vermersch, P. The prevalence of Sjögren syndrome in patients with primary progressive multiple sclerosis. Neurology 2001, 57, 1359-1363. [CrossRef] [PubMed] 